\title{
RATE CONTROL FOR THERAPEUTIC APPLICATIONS IN INTERNET OF BIO-NANO THINGS USING MOLECULAR COMMUNICATION: A SURVEY
}

\author{
Shirin Salehi ${ }^{1}$, Naghmeh Sadat Moayedian ${ }^{2}$, Mohammad Taghi Shafiee $^{1}$ \\ ${ }^{1}$ Communications Regulatory Authority, Ministry of ICT, Iran, ${ }^{2}$ Department of Electrical and Computer Engineering, \\ Isfahan University of Technology, Isfahan 84156-83111, Iran
}

NOTE: Corresponding author: Naghmeh Sadat Moayedian, moayedian@cc.iut.ac.ir

\begin{abstract}
Molecular communication is transmitting and receiving chemical signals using molecules and is an interdisciplinary field between nanotechnology, biology, and communication. Molecular communication can be used for connecting bio-nano things. The connected nano-things build a nano-network. Transport mechanisms in molecular communication include free diffusion, gap junction channels, molecular motors, self-propelling microorganisms like bacteria and random collision of mobile nano-things. Free diffusion is the most widely used transport mechanism in the literature. Brownian motion is always available and its energy consumption is zero. This paper explores the therapeutic applications of rate control in the Internet of Bio-Nano Things and reviews the recent trends and advancements in the field of molecular communication. These methods aim to guarantee the desired rate of drug molecules at the target site and overcome the side effects of excessive emission.
\end{abstract}

Keywords - Bio-nano things, molecular communication, release rate control, targeted drug delivery

\section{INTRODUCTION}

Nanomachines are the most basic operational units with dimensions of several hundred nanometers to a maximum of a few micrometers and have limited capabilities. They can perform simple tasks such as sensing, stimulating, calculating and storing information. In order to increase the capabilities of nanomachines and use them in real scenarios, a network must be formed between them. In this way, nanomachines are able to collaborate, combine or share information. The connection of these nanomachines is called a nano-network [1]. In fact, nanonetworks are a new research branch that results from the use of nanotechnology in the field of digital communications [2, 3]. The very small size of nanomachines makes the physical telecommunication channels in nanonetworks significantly different from traditional wireless and wired channels. Currently, there are two methods for connecting nanomachines: nano-electromagnetic communication and molecular communication [1].

Nano-electromagnetic communication is the sending and receiving of electromagnetic waves between nanoscale components. There is ambiguity about how nanoantennas can be achieved by shrinking existing antennas. The frequency released by the antenna comes from the $f \propto \frac{v}{L}$, in which $v$ is wave propagation speed and $L$ is antenna length, so in the expected size of a nanomachine, the frequency released by nano-antenna will be in the optical range (hundreds of $\mathrm{THz}$ ). Although this frequency leads to a lot of bandwidth, it will also have a lot of loss, which causes the range of these nano-antennas to be almost zero, so traditional electromagnetic telecommunication methods must be deeply revised before using them in new scenarios [4].
To overcome these limitations, graphene-based nanoantennas are provided. Graphene is a form of carbon composed of flat sheets with the thickness of an atom in which atoms are placed as honeycomb lattices. Due to its specific properties, graphene-based nano-antennas will radiate at a lower frequency than the Terahertz band, and the channel attenuation will be much less at this frequency, therefore, graphene-based nano-antennas allow for nanoscale electromagnetic communication $[5,6]$.

Molecular communication is a new method that is inspired by the communication between living cells. In molecular communication, information is transmitted through message molecules [7]. The advantages of this solution compared to nano-electromagnetic communication are inherent nanoscale, biocompatibility and low energy consumption [8]. In molecular communication, chemical signals or molecules are sent and received. There are many differences between molecular communication and traditional communication: In molecular communication, the message is encoded in molecules, whereas in traditional networks, information is encoded in electromagnetic, audio and optical signals. The rate of wave propagation in traditional networks is much faster than the speed of the propagation of molecular messages. In addition, in molecular communication, most of the energy consumed is chemical and power consumption is low, while electrical energy is used in traditional networks.

Molecular communication can be effective in medical applications due to their biocompatibility $[9,10,11,12]$. The components of molecular communication are: transmitting nanomachine, receiving nanomachines, messenger molecules, interface molecules and transport mecha- 
nisms [13]. There are five transport mechanisms which include free diffusion, gap junction channels, molecular motors, self-propelling microorganisms and random collision of nanomachines. Molecular communication systems can be classified with various criteria as shown in Fig. 1:

a) Scale: The molecular communication scale refers to the distances at which bio-nanomachines interact with each other by releasing molecules and is divided into three categories: intracellular, intercellular and inter-organ [14]. Similarly, bio-nanomachines are divided into three categories, short-range such as molecular motors, mediumrange such as ion signaling and transmission through diffusion, and finally long-range transmission such as transmission based on bacteria, neurons and pheromone signaling [7]. Pheromone is a chemical agent secreted or excreted, leading to the stimulation of a collective response in members of a species.

b) Energy consumption: Molecules are either dispersed in the environment or released directionally by chemical energy consumption, which is called Passive Molecular Communication (PMC) and Active Molecular Communication (AMC) $[15,14]$, respectively.

c) Communication type: Molecular communications can be divided into two categories: wired and wireless.

i) Wired: Refers to methods that require a physical link to transmit signals [8] and they are also called Walkwaybased methods [16]. For example, microtubules are provided in micro-dimensions to connect nanomachines. Data transfer between nanomachines is done through the movement of molecular motors that walk on microtubules. Another example of wired molecular communication is neuronal messaging that is placed in the category of inactive molecular communication [17].

ii) Wireless: Wireless methods refer to methods that require only a fluidic medium (such as air, water and blood) without the need for electrical conductors or physical links to transmit information [8]. Most current molecular communication methods mimic wireless communication samples. This is due to the similarity of natural biological communication systems with wireless communication systems. Of course, the fundamental difference is that the distribution of molecules is slow compared to the propagation of radio waves. One example of this method is calcium signaling. In this method, the propagation of waves between cell junctions is carried out in densely packed cell tissue. Calcium waves are commonly used to transmit short-range information between cells where information is coded in the concentration of calcium ions. Another example of wireless data transmission is the use of bacteria [17]. The wireless method can be divided into two categories: A) Flow-based: In this method, molecules are released through diffusion in a fluidic medium such as blood flow. Intra-body hormonal communication is the most important example of this method [16]. B) Diffusion-based: In this method, molecules are propagated by diffusion in a fluidic medium and molecules only follow the rules of random walk. Pheromonal communi- cations, calcium signaling and quorum sensing in bacteria are examples of this method [16]. Quorum sensing is a population-proportional stimulation and response system that bacteria use to coordinate with each other.

Medical applications, such as Targeted Drug Delivery (TDD), activation of the immune system and tissue engineering, are one of the most important application categories of molecular communication. In such scenarios, rate control mechanisms play an essential role. In TDD, rate control is indispensable to maintain the drug concentration between the Least Effective Concentration (LEC) and the Maximum Tolerated Concentration (MTC). Moreover, bio-nanomachines generally bear a limited resource, therefore releasing the drug molecules at the optimal rate is of significant importance. Controlling the rate of activators or antagonists is also indispensable in the immune system activity scenario i.e., to intensify or reduce the activity of the immune system. The rate control is required as well in tissue engineering scenarios to ensure the prolonged exposure of the damaged tissue to the growth factors. Considering the importance of rate control in therapeutics applications, this paper aims to review the state-of-the-art literature on rate control using molecular communication.

The organization of this paper is as follows: Rate control in molecular communication with a layer architecture perspective is investigated in Section 2. Section 3 presents the therapeutic applications of rate control. Section 4 reviews the recent advancements and current research on releasing rate control in DDS. Research opportunities are presented in Section 5. Section 6 concludes this paper.

\section{RATE CONTROL IN MOLECULAR COMMU- NICATION}

Molecular communication architecture can be examined from the perspective of communication networks $[18,14$, 19]: molecular physical layer, molecular link layer, molecular network layer, molecular transport layer and molecular application layer. Since our paper is focused on rate control in molecular communication, we review the existing works in the transport layer. This layer provides the functions needed for end-to-end transmission and is responsible for providing reliability, In-sequence delivery, congestion control and flow control. Rate control in molecular communication is carried out for two purposes: Flow control and congestion control.

\subsection{Flow control}

Flow control is a function in which the sender adjusts the transmission rate of the molecular transmission layer based on the receiver's characteristics. Flow control can be started by the sender or receiver [19]:

i) Transmitter initiated flow control: The easiest form of flow control is that the sender uses a very low transmission rate so that there are no losses. This requires prior 


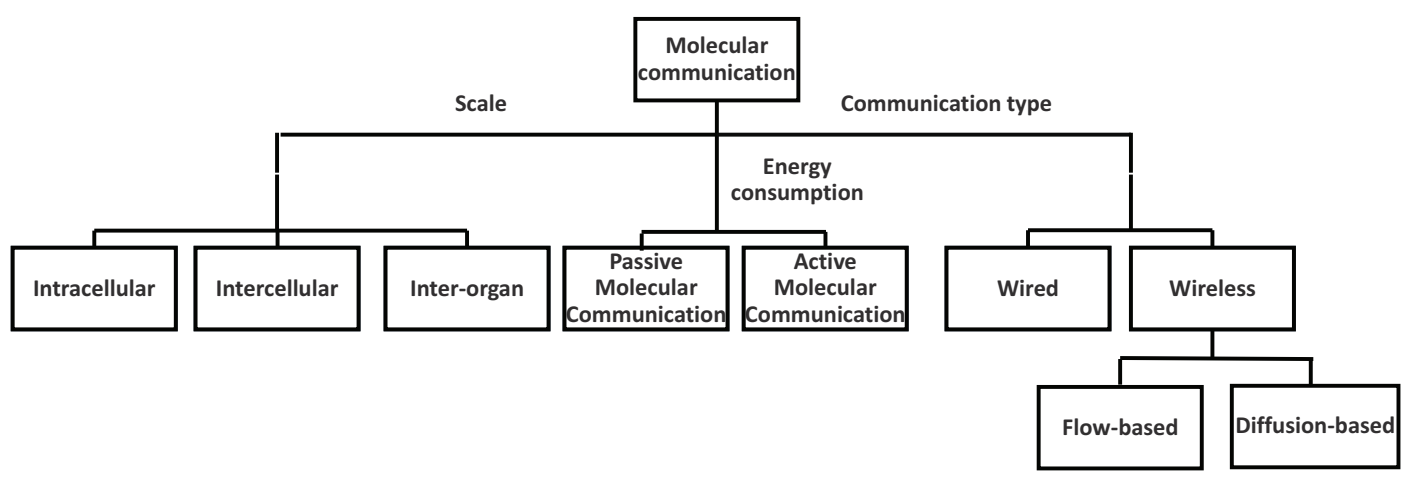

Fig. 1 - Classification of molecular communication based on scale, energy consumption and communication type

knowledge of the recipient's processing capabilities. A more complex shape requires the transmitter to be able to detect the losses of molecular frames. The transmitter may feel the existence or absence of expected chemical reactions in the receiver in the environment and adjust the sending rate accordingly.

ii) Receiver-initiated flow control: When the recipient's molecular storage is filling up, the receiver informs the transmitter by sending feedback. This form of flow control is commonly used in traditional communication. So far, only one method of flow control initiated by the receiver has been studied in $[20,18]$. In order to control the flow of information in traditional networks, the recipient announces a window size to the sender, meaning that the unacknowledged number of bytes of the sender should not exceed that value at any given moment. If the recipient reads the information as fast as it reaches, the window will remain open, otherwise, the window size will be reduced by reaching each segment to eventually reach zero. In [18], $N$ senders and $M$ receivers are considered. First, an optimization problem is proposed to find the maximum throughput and efficiency according to the sender's transmission rate. Throughput is the number of molecules processed by receptors per unit time. Efficiency is throughput divided by the number of molecules sent per unit time. For simplicity, it is assumed that all transmitters are located at the origin and all receivers are located at a similar location. With this assumption, a mathematical expression is obtained for the upper bound of throughput and efficiency. Then, the optimal transmission rate that maximizes them is calculated numerically. It can be seen that there is a compromise between throughput and efficiency. In the following, [18] presents a method for controlling the transmission rate using positive and negative feedback. In this method, the receiver bio-nanomachine releases feedback molecules in the environment in response to the transmitter. The transmitter bio-nanomachine then regulates its transmission rate based on the concentration of feedback molecules. In the Negative Feedback (NF) the transmitter bio-nanomachine reduces the transmission rate by increasing the concentration of feedback molecules. In the Positive Feedback (PF), the opposite is true. Simulation results show that the negative feedback-based method provides maximin strategy by changing the number of transmitters, number of receivers, molecule degradation rate, diffusion coefficient and location, i.e. maximizes the minimum throughput. The positive feedback method is also a maximin strategy for efficiency by changing the number of receivers, diffusion coefficient and degradation rate of molecules, i.e. maximizes the minimum efficiency.

In [12], a TCP-like protocol is proposed to find the optimal transmission rate between the transmitter and receiver and prevent congestion in molecular communication. In this method, the transmitter is assumed to be very simple and the receiver acts as a control node and sends the connection signal to the transmitter. This triggers the transmitter to release molecules. The transmitted molecules are released in the environment and are absorbed by the receptors on the receiver surface. When the receiver absorbs the desired amount of the transmitter molecules, it releases a disconnection signal to prevent the transmitter from continuing the transmission. Similar to the TCP transmitter, which is not already aware of the maximum network capacity, the transmitter first increases the transmission rate. In TCP, this increase is done exponentially in the first step and in the stage of avoiding congestion linearly with the round trip time. In this scenario, for simplicity, only a linear rate increase is considered. Then, the receiver decides to halve or stop the transmission rate according to the round trip time.

\subsection{Congestion control}

Congestion control is used to regulate the number of molecular transfer data units in order to prevent congestion in the middle nodes. This can be done by setting the sender's transmission rate. By detecting an error, the sender's transmission rate can be reduced to a specified predetermined value. It is also possible to calculate the new transmission rate according to the amount of congestion. Congestion can be detected from the error rate. After the rate decreases, the sender may start to increase the sending rate again so that it can use the maximum channel capacity. The amount of this increase can be constant or 
depends on a network parameter (e.g. how long the error hasn't occurred), in which case remembering the history is necessary.

In the past 30 years, the issue of congestion control in packet networks has been considered by many researchers. However, little attention has been paid to this issue in molecular networks [21]. In [21], congestion is defined as the inability of the receiver to accept all molecules in the receiver space and the congestion control problem has been investigated in a drug delivery scenario. This limitation occurs for two reasons: limited number of receptors at the receiver surface and significant trafficking time. Trafficking time is defined as the time required for a ligand to bind a receptor plus the time needed to internalize the complex. In [21], theoretical analysis of congestion reasons in a diffusion-based molecular communication is carried out and a receiving model including a set of queue systems is presented.

\section{THERAPEUTIC APPLICATIONS OF RATE CONTROL}

\subsection{Targeted drug delivery}

In drug delivery applications, if the transmitter sends molecules at a higher rate than what the receiver can process, these molecules will disperse in the environment and can lead to adverse side effects in other areas. Also, since drug molecules are expensive in some cases, it is important to prevent the loss of these molecules.

Molecular communication enables new methods of drug delivery by creating cooperation between nanomachines. For example, a large number of bio-nanomachines can be injected into a patient's body to perform massively parallel searches of the diseased site. When the bionanomachine detects the signal molecule secreted by the disease site, it will amplify the signal molecule, thereby increasing the concentration of the signal molecule in the environment, causing more bio-nanomachines to reach the disease site. A group of bio-nanomachines at the target site can also communicate via quorum sensing to estimate the number of bio-nanomachines in the environment and increase (or decrease) the rate of drug release if the number of nanomachines is small (or large) to achieve the continuous release of drug molecules into the environment. A group of bio-nanomachines with different functions can also be used to detect different environmental conditions, communicate to aggregate sensed conditions, and perform complex calculations such as logic calculations to determine whether drug molecules are released [14]. Another example of collaboration between nanomachines using molecular communication is encapsulated drug transmitters that help to increase the lifetime of a drug delivery system and solve the problem of limited reservoir capacity, as described in [22]. These methods are designed to maximize the therapeutic effects of drug molecules; therefore, a group of nanomachines must communicate with each other at the target site to adjust the rate of release of drug molecules based on different conditions, such as the spatial distribution of target cells and bio-nanomachines.

TDD enables local drug delivery, which is not possible with systemic drug delivery. By placing the drug delivery system near the tumor site, the drug is released only at the site of the disease and the drug level is low in other sensitive areas of the body [23]. On the other hand, other drug delivery systems that are injected intravenously may be rapidly excreted by cleansing organs such as the kidneys, which can lead to healthy tissue being exposed to toxic levels of the drug. To address these problems, local methods such as intra-tumor injection and drug release in the vicinity of unhealthy tissue have been studied, including $[24,25]$ to treat skin cancer and [26] for the treatment of bladder cancer.

\subsection{Immune system}

Rate control can also be used to activate the immune system. The immune system is made up of different types of cells that are able to perform specific functions against pathogens and threats. Each type of immune cell specializes in fighting a specific threat and is stimulated by a sequence of message molecules that are released by the immune cells responsible for detecting pathogens. The immune system is stimulated to kill bacteria or viruses, heal diseased cells, and eradicate cancerous tumors. Molecular communication and artificial nanomachines can stimulate the immune system and be used to intensify the response to a variety of threats [11]. In some cases, it is also necessary to prevent the immune system from responding by releasing molecules that can reduce the activity of the immune system. Synthetic antagonists can also be used to reduce the immune response. Therefore, similar to TDD, there is a need to monitor and control the rate of activators or antagonists.

\subsection{Tissue engineering}

Another application of molecular communications where the rate control is required is tissue engineering [14, 11 , 27]. Tissue engineering can lead to organ construction and help patients with tissue or organ failure. By placing nanomachines in engineered organs, intelligent organs can be produced that can diagnose diseases.

Tissue engineering has a role in regenerative medicine. The goal of regenerative medicine is the replacement of damaged tissues and organs by using transplanted cells at the injured site. Cellular signaling plays an essential role in development of the tissue. This signaling is often based on diffusion and direct cell-to-cell interactions. Under such conditions, molecules such as peptides and proteins regulate the absorption of soluble growth factors, and the adhesion, migration, proliferation and differentiation of a large number of cell types. All these factors effectively control the response of the cells and ultimately the formation of the new tissues. 
There is no doubt that growth factors are essential for the control of a multitude of biological processes, such as tissue regeneration; but these factors have a short life; because they are eliminated in the body immediately after release. The success of tissue regeneration depends on how long the tissue has been exposed to these factors. The highest rate of regeneration is achieved when the tissue is exposed to these factors throughout the whole repair process. For this purpose, a set of controlled delivery systems is considered that is able to release growth factors so that the tissue is continuously and for a long time exposed to such factors with an almost constant concentration [11]. Coordination between the nanomachines responsible for this operation can be done by molecular communication; In this way, the release and control of the place and time of growth factors can be done accurately. In this scenario, we need rate control methods as well to control the rate of growth factors.

\section{CURRENT RESEARCH ON RELEASING RATE CONTROL IN LOCAL DDS}

When the goal of delivering a drug at a target site like a tumor is considered, the drug should be released at an appropriate rate in such a way that the level of the drug is kept within the therapeutic limits during the duration of treatment [28]. In a sustained drug release system, nanotransmitters release drug molecules over a relatively long period of time to ensure long-term treatment of the target area. In this treatment method, the concentration of the drug should be placed between the two values of LEC and the MTC.

If the concentration of the drug at the target site is lower than the LEC, the drug is not effective enough, and if it is higher than the MTC, the drug can have harmful effects on healthy parts [29]. Rate control in this way can be used in other applications as well, such as tissue engineering, to control growth factors rates or to control the rate of activators in activating the immune system.

In recent years a great deal of research has been devoted to showing the application of molecular communication in order to enhance the efficiency of a TDD system by controlling the release rate of bio-nanomachines. A comparison of state-of-the-art literature on releasing rate control for TDD is shown in Table 1. The number of required type of molecules, nanomachine mobility, nanomachines configuration, receiver type and the transport mechanism are represented in this table.

To achieve this goal, in [30], a local multi-nanotransmitter drug delivery system at a constant rate is formulated as an image processing problem, and the minimum density required to get enough medicine to all parts of an arbitrary tumor of a certain size is obtained. The effect of distribution of nanomachines on system performance is also investigated. In this case, only the nano-transmitters located at the target site are activated. Therefore, the drug is confined to the target site and is not propagated in healthy parts of the body.
In [31], a single and multiple transmitter local drug delivery system with limited resources is designed at an optimal release rate. In this case, the nearest transmitters to a randomly located tumor are activated to provide the least effective concentration at the target site. In this scenario, the optimal rate of transmitter nanomachines is determined in such a way that the total release rate is minimized provided that the least effective concentration is available at the target site. Drug nano-transmitters have limited resources in terms of energy and reservoir and these limitations should be taken into account when designing a drug delivery system. Also, drug molecules may be expensive, and releasing a large number of them can lead to damage to healthy parts of the body.

A controlled-release drug delivery system with mobile drug carrier and absorbing receiver is proposed in [32, 33]. Mobile drug carriers are considered as mobile transmitters, the targeted disease cells as absorbing receiver and the channel between the transmitter and receiver as a time-variant channel. This leads to a time-dependent release rate of drug molecules. Numerical results show that the mobile transmitter controlled-release drug delivery system outperforms constant-release rate design.

In [34], the problem of joint optimization of molecules allocation and relay location is considered to minimize the error probability. Molecule allocation is necessary because of the finite availability of molecule synthesizing energy and limited storage capabilities of the reservoir.

Another release rate optimization problem is suggested in [35]. In this paper, an optimization problem is formulated to optimize the number of transmitters and transmit power such that the drug concentration at the receiver site is kept above LEC, while the interference at other receivers is maintained below the MTC threshold. The path loss of the human circulatory system is also taken into account.

Authors in [36] proposed a drug release rate optimization based on $\mathrm{M} / \mathrm{M} / \mathrm{c} / \mathrm{c}$ queue for the purpose of local drug delivery. Drug reception model in this paper is based on $\mathrm{M} / \mathrm{M} / \mathrm{c} / \mathrm{c}$ queue to simulate the interactions between ligands and receptors. The optimal release rate is derived from the LEC.

A drug release synchronization issue in a multiple transmitter local DDS is addressed in [37]. It is assumed that a trigger source transmits a signal to the transmitting nanomachines to initiate the drug release. However, the propagation delay causes the nanomachines to release molecules in a nonsimultaneous manner. The aim of this work is to minimize the release-time error considering the propagation delay.

In [38], the impact of feedback control is investigated in an Amplitude Shift Keying (ASK)-based Molecular Communication (MC) system, which is of great importance in DDS. In this paper, a one-dimensional channel with drift velocity caused by blood flow is considered. The receiver is assumed to be absorbing with a limit on ligand-receptor binding due to saturation. The input is limited by a toxicity constraint of injected molecules. It is shown that, 
Table 1 - Comparison of state-of-the-art research on releasing rate control for TDD

\begin{tabular}{|c|cccccc|}
\hline Reference & Year & $\begin{array}{c}\text { Required } \\
\text { type of } \\
\text { molecule }\end{array}$ & $\begin{array}{c}\text { Nanomachines } \\
\text { Mobility }\end{array}$ & $\begin{array}{c}\text { Nanomachines } \\
\text { configuration }\end{array}$ & Receiver type & $\begin{array}{c}\text { Transport } \\
\text { mechanism }\end{array}$ \\
\hline$[29]$ & 2016 & 1 & Static & MISO & Transparent & Diffusion \\
\hline$[30]$ & 2017 & 1 & Static & MISO & Transparent & Diffusion \\
\hline$[31]$ & 2019 & 1 & Dynamic & SISO & Absorbing & Diffusion \\
\hline$[32]$ & 2019 & 1 & Dynamic & SISO & Absorbing & Diffusion \\
\hline$[33]$ & 2018 & 2 & Static & SISO & Absorbing & Diffusion \\
\hline$[34]$ & 2018 & 1 & Static & MIMO & - & Diffusion-drift \\
\hline$[35]$ & 2020 & 1 & Static & SISO & Absorbing & Diffusion \\
\hline$[36]$ & 2020 & 1 & Static & MISO & Absorbing & Diffusion \\
\hline$[37]$ & 2021 & 1 & Static & SISO & Absorbing & Diffusion-drift \\
\hline$[38]$ & 2018 & 2 & Static & MISO & Transparent & Diffusion \\
\hline$[39]$ & 2020 & 1 & Dynamic & MISO & Absorbing & Diffusion-drift \\
\hline$[40]$ & 2019 & 3 & Dynamic & MISO & - & Diffusion \\
\hline
\end{tabular}

especially for higher values of toxicity constraint and sequence length, feedback, in terms of causal knowledge of the number of delivered molecules, improves performance of ASK-based molecular communication.

In [22], a multiple transmitter local drug delivery system associated with encapsulated drug transmitters is investigated in order to overcome one of the limitations of drug delivery systems, the reservoir capacity. In order to improve the lifetime of drug transmitting nanomachines, and, hence, the longevity of a drug delivery scenario, the system is associated with encapsulated drug transmitters. Encapsulated drugs are incapable of reaction with the environment unless they are unpacked in a drug transmitter nanomachine. Therefore, far-reaching transmitters do not have harmful effects on the healthy parts of the body. The advantage of this protocol is to increase the time interval between consecutive administrations without increased toxicity. As a result, it improves the mental health of patients and reduces the costs of treatment. The lifetime of this drug delivery system depends on the distribution and topology of encapsulated drug transmitters rather than their rates. Finally, a lower bound is derived on the expected lifetime of a Poisson distributed random network of nanomachines. The performance of this lifetime improvement protocol is compared with two other protocols which are the direct extension of the drug delivery system introduced in [31].

A TDD system with intelligent nanomachines which does not only rely on blood vessel circulation, is presented in [39]. A big intelligent nanomachine takes small intelligent nanomachines and drugs to the vicinity of the tumor area to release drug molecules there. The nanomachines are assumed to be resource constrained with simple intelligence. They collaborate to find the path between the home and destination. They then go back to the home, load the drug again and repeat the delivery process.

In[40], a single receptor is modeled as an $M / M / 1 / 1$ queue considering the complementary blocking probability and the mean service time. Therefore, the stochastic nature of ligand-receptor binding, which comes from the incapability of a receptor to receive all molecules in its space; and also known as receptor occupancy is modeled. These findings can have a crucial role in designing drug delivery systems in which determining the optimal rate of the drug transmitting nanomachines is critical to avoid toxicity while maintaining effectiveness.

\section{RESEARCH OPPORTUNITIES}

Researchers are continuously exploring ideas to provide enhanced healthcare delivery in ways that either complements existing solutions or introduce entirely new technological solutions. In recent years, considerable research in the area of molecular communication has been devoted to design and implement a more effective drug delivery system. Considering the current research and advancements in the field of TDD using molecular communication, a number of research opportunities are suggested as follows:

1. Considering the dynamic conditions of the environment and the tumor in the TDD system so that the amount of drug released can be controlled according to the environmental conditions, size and activity of the tumor. The optimal drug delivery rate calculated in many works is with the assumption of stable environmental conditions and tumor characteristics. If the environmental conditions or characteristics of the tumor change, the drug delivery rate of the transmitter nanomachines should be adjusted accordingly automatically or by applying an external stimulus.

2. Investigating the rate control problem in similar applications such as tissue engineering and rate control of growth factors: Although the rate control problem in tissue engineering applications is very similar to rate control in a TDD system, details of the new problem should be considered in rate control system design. 
3. Considering reflective spherical nano-transmitters instead of point nano-transmitters for directional gain in the TDD scenario: Having a directional gain can increase the gain of the drug delivery system and prevent the molecular dispersion in non-target environments.

\section{CONCLUSION}

Molecular communications is a promising paradigm to exchange information among bio-nanomachines and is realized through transmission, propagation and eventually reception of molecules. It is known as a practical solution for communication among nano-networks. This idea is inspired by nature and by observing successful molecular transmissions inside cells and between cells.

The transmission mechanism which is widely assumed in the related literature is free diffusion. Free diffusion is the most basic transmission mechanism in molecular communication. The advantages of this mechanism are zero energy consumption and no need for infrastructure. Of course, this method requires a large number of molecules and due to the random movement of molecules, the time needed to reach the destination is high.

In this paper, releasing rate control for therapeutic applications such as drug delivery using molecular communication is considered. In stable drug delivery systems, nano-transmitters must release drug molecules over a relatively long period of time to ensure target treatment. Therefore, transmitter nanomachines should use continuous release instead of instantaneous release of drug molecules. Also, the concentration of the drug at the target site should be between two amounts of minimum effective concentration and maximum tolerable concentration. Rate control is used in other applications such as tissue engineering and the immune system.

\section{REFERENCES}

[1] I F Akyildiz, F Brunetti, and C Blázquez. "Nanonetworks: A new communication paradigm". In: Computer Networks 52.12 (2008), pp. 2260-2279. ISSN: 13891286.

[2] Hoda ShahMohammadian. "System design for nano-network communications". $\mathrm{PhD}$ thesis. University of Calgary, 2013.

[3] Stephen F Bush. Nanoscale communication networks. Artech House, 2010.

[4] Ignacio Llatser. "Exploring the Scalability Limits of Communication Networks at the Nanoscale". PhD thesis. Universitat Politecnica de Catalunya, 2013, p. 182.

[5] Ian F Akyildiz, Josep Miquel Jornet, and Massimiliano Pierobon. "Propagation models for nanocommunication networks". In: Antennas and Propagation (EuCAP), 2010 Proceedings of the Fourth European Conference on. IEEE. 2010, pp. 1-5.
[6] Josep Miquel Jornet and Ian F Akyildiz. "Graphenebased nano-antennas for electromagnetic nanocommunications in the terahertz band". In: Antennas and Propagation (EuCAP), 2010 Proceedings of the Fourth European Conference on. IEEE. 2010, pp. 1-5.

[7] Mehmet S Kuran, Huseyin Birkan Yilmaz, Tuna Tugcu, and Ian F Akyildiz. "Modulation techniques for communication via diffusion in nanonetworks". In: Communications (ICC), 2011 IEEE International Conference on. IEEE. 2011, pp. 1-5.

[8] Lluís Parcerisa Giné and Ian F Akyildiz. "Molecular communication options for long range nanonetworks". In: Computer Networks 53.16 (2009), pp. 2753-2766.

[9] Ian F Akyildiz, Josep Miquel Jornet, and Massimiliano Pierobon. "Nanonetworks: A new frontier in communications". In: Communications of the ACM 54.11 (2011), pp. 84-89.

[10] Nariman Farsad, H Birkan Yilmaz, Andrew Eckford, Chan-Byoung Chae, and Weisi Guo. "A comprehensive survey of recent advancements in molecular communication". In: IEEE Communications Surveys \& Tutorials 18.3 (2016), pp. 1887-1919.

[11] L. Felicetti, M. Femminella, G. Reali, and P. Liò. "Applications of molecular communications to medicine: A survey". In: Nano Communication Networks 7 (2016), pp. 27-45. ISSN: 18787789.

[12] Luca Felicetti, Mauro Femminella, Gianluca Reali, Tadashi Nakano, and Athanasios V Vasilakos. "TCPlike molecular communications". In: IEEE Journal on Selected Areas in Communications 32.12 (2014), pp. 2354-2367.

[13] Baris Atakan. Molecular Communications and Nanonetworks. Springer, 2016.

[14] Tadashi Nakano, Andrew W Eckford, and Tokuko Haraguchi. Molecular communication. Cambridge University Press, 2013.

[15] Baris Atakan, Ozgur B Akan, and Sasitharan Balasubramaniam. "Body area nanonetworks with molecular communications in nanomedicine". In: IEEE Communications Magazine 50.1 (2012).

[16] M Pierobon and I F Akyildiz. "Fundamentals of Diffusion-Based Molecular Communication in Nanonetworks". PhD thesis. Georgia Institute of Technology, 2013, pp. 1-147.

[17] Sasitharan Balasubramaniam, Sigal Ben-Yehuda, Sophie Pautot, Aldo Jesorka, Yevgeni Koucheryavy, et al. "A review of experimental opportunities for molecular communication". In: Nano Communication Networks 4.2 (2013), pp. 43-52. 
[18] Tadashi Nakano and Yutaka Okaie. "Transmission Rate Control for Molecular Communication among Biological Nanomachines". In: IEEE Journal on Selected Areas in Communications 31.12 (2013), pp. 835-846.

[19] Tadashi Nakano, Tatsuya Suda, Yutaka Okaie, Michael John Moore, and Athanasios V. Vasilakos. "Nanomachines : A Layered Architecture and". In: IEEE Transactions on Nanobioscience 13.3 (2014), pp. 169-197.

[20] Tadashi Nakano, Yutaka Okaie, and Athanasios V Vasilakos. "Throughput and efficiency of molecular communication between nanomachines". In: Wireless Communications and Networking Conference (WCNC), 2012 IEEE. IEEE. 2012, pp. 704-708.

[21] Mauro Femminella, Gianluca Reali, and Athanasios V. Vasilakos. "Molecular communications model for drug delivery". In: IEEE Transactions on Nanobioscience 14.7 (2015), pp. 935-945. ISSN: 15361241.

[22] Shirin Salehi, Naghmeh S Moayedian, Shaghayegh Haghjooy Javanmard, and Eduard Alarcón. "Lifetime improvement of a multiple transmitter local drug delivery system based on diffusive molecular communication". In: IEEE transactions on nanobioscience 17.3 (2018), pp. 352-360.

[23] Narayan Bhattarai, Jonathan Gunn, and Miqin Zhang. "Chitosan-based hydrogels for controlled, localized drug delivery". In: Advanced drug delivery reviews 62.1 (2010), pp. 83-99.

[24] Abhijit Ray, Matthew A Williams, Stephanie M Meek, Randy C Bowen, Kenneth F Grossmann, Robert HI Andtbacka, Tawnya L Bowles, John R Hyngstrom, Sancy A Leachman, Douglas Grossman, et al. "A phase I study of intratumoral ipilimumab and interleukin-2 in patients with advanced melanoma". In: Oncotarget 7.39 (2016), p. 64390.

[25] Praveen K Bommareddy, Ann W Silk, and Howard L Kaufman. "Intratumoral approaches for the treatment of melanoma". In: The Cancer Journal 23.1 (2017), pp. 40-47.

[26] Shruti GuhaSarkar, Prachi More, and Rinti Banerjee. "Urothelium-adherent, ion-triggered liposome-in-gel system as a platform for intravesical drug delivery". In: Journal of Controlled Release 245 (2017), pp. 147-156.

[27] Prakriti Tayalia and David J Mooney. “Controlled growth factor delivery for tissue engineering". In: Advanced Materials 21.32-33 (2009), pp. 32693285 .

[28] T. M. Allen. "Drug Delivery Systems: Entering the Mainstream". In: Science 303.5665 (2004), pp. 1818-1822. ISSN: 0036-8075. DOI: $10.1126 /$ science. 1095833.
[29] Juergen Siepmann, Ronald A Siegel, and Michael J Rathbone. Fundamentals and applications of controlled release drug delivery. Springer US, 2012.

[30] Shirin Salehi, Simon S Assaf, Raul G Cid-Fuentes, Naghmeh S Moayedian, Josep Solé-Pareta, and Eduard Alarcón. “Optimal deployment of multiple transmitter drug delivery system: A spatial sampling theorem approach". In: Proceedings of the 3rd ACM International Conference on Nanoscale Computing and Communication. 2016, pp. 1-2.

[31] Shirin Salehi, Naghmeh S Moayedian, Simon S Assaf, Raul G Cid-Fuentes, Josep Solé-Pareta, and Eduard Alarcón. "Releasing rate optimization in a single and multiple transmitter local drug delivery system with limited resources". In: Nano Communication Networks 11 (2017), pp. 114-122.

[32] Trang Ngoc Cao, Arman Ahmadzadeh, Vahid Jamali, Wayan Wicke, Phee Lep Yeoh, Jamie Evans, and Robert Schober. "Diffusive mobile MC for controlled-release drug delivery with absorbing receiver". In: ICC 2019-2019 IEEE International Conference on Communications (ICC). IEEE. 2019, pp. 1-7.

[33] Trang Ngoc Cao, Arman Ahmadzadeh, Vahid Jamali, Wayan Wicke, Phee Lep Yeoh, Jamie Evans, and Robert Schober. "Diffusive mobile MC with absorbing receivers: Stochastic analysis and applications". In: IEEE Transactions on Molecular, Biological and Multi-Scale Communications 5.2 (2019), pp. 84-99.

[34] Satish K Tiwari, Tadi Ravi Teja Reddy, Prabhat K Upadhyay, and Daniel Benevides Da Costa. "Joint optimization of molecular resource allocation and relay positioning in diffusive nanonetworks". In: IEEE Access 6 (2018), pp. 67681-67687.

[35] Keishla D Ortiz-Lopez, Mahima Agumbe Suresh, and Radu Stoleru. "Transmitters location optimization for drug delivery systems". In: Proceedings of the 5th ACM International Conference on Nanoscale Computing and Communication. 2018, pp. 1-6.

[36] Qingying Zhao and Min Li. "Release Rate Optimization Based on $\mathrm{M} / \mathrm{M} / \mathrm{c} / \mathrm{c}$ Queue in Local Nanomachine-Based Targeted Drug Delivery". In: International Conference on Bio-inspired Information and Communication Technologies. Springer. 2020, pp. 130-140.

[37] Tania Islam, Ethungshan Shitiri, and Ho-Shin Cho. "A Simultaneous Drug Release Scheme for Targeted Drug Delivery Using Molecular Communications". In: IEEE Access 8 (2020), pp. 91770-91778.

[38] Siavash Ghavami, Raviraj Adve, and Farshad Lahouti. "Information rate analysis of ASK-based molecular communication systems with feedback". In: Nano Communication Networks 28 (2021), p. 100339. 
[39] Lin Lin, Fupeng Huang, Hao Yan, Fuqiang Liu, and Weisi Guo. "Ant-behavior inspired intelligent nanonet for targeted drug delivery in cancer therapy". In: IEEE transactions on nanobioscience 19.3 (2020), pp. 323-332.

[40] Shirin Salehi, Naghmeh S Moayedian, and Eduard Alarcón. "Diffusion-Based Molecular Communication Channel in Presence of a Probabilistic Absorber: Single Receptor Model and Congestion Analysis". In: IEEE transactions on nanobioscience 18.1 (2019), pp. 84-92.

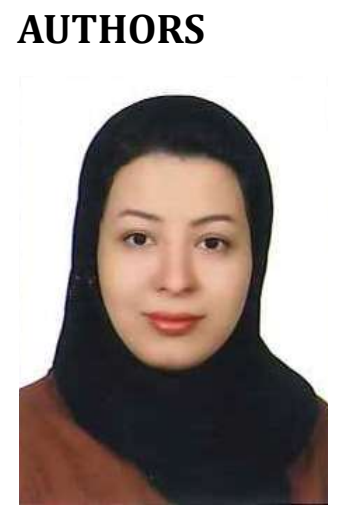

Shirin Salehi received a Ph.D. degree in electrical engineering, communication systems from the Isfahan University of Technology (IUT), Iran, in 2018. She was a visiting scholar with the NaNoNetworking Center in Catalunya (N3Cat), Universitat Politècnica de Catalunya, Spain, from 2016 to 2017. From 2018 to 2019, she was a Postdoctoral Researcher with the ECE Department, Isfahan University of Technology. Her research interests include cooperative communication systems, molecular communications with application in a medical field, and digital signal processing. She is currently affiliated with the communication regulatory authority, ministry of ICT, Iran.

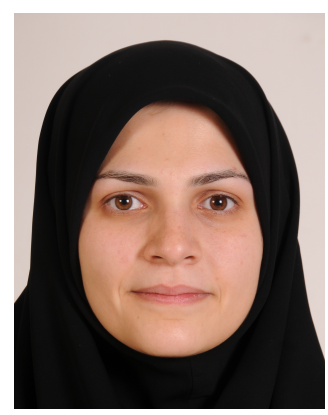

Naghmeh Sadat Moayedian received B.S., M.S., and Ph.D. degrees in electrical engineering from the Isfahan University of Technology, Isfahan, Iran, in 1999, 2002, and 2009, respectively. She is currently affiliated with the ECE Department, Isfahan University of Technology. Her research interests include cloud networks, nano-networks, and wireless networks.

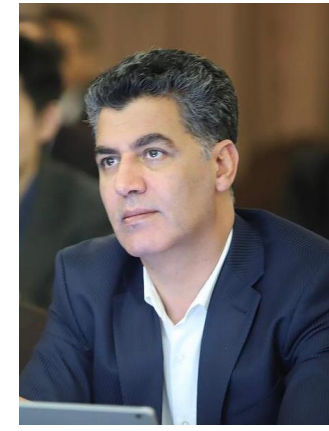

Mohammad Taghi Shafiee received a Ph.D. degree in human resources management from the Shakhespajouh Research Institute, University of Isfahan, Iran, in 2016 and M.Sc. degree in electrical engineering, communication systems from the K.N. Toosi University of Technology, Iran, in 1995. Since 1995, he has

participated and contributed in various international events of of the International Telecommunication Union and Asia-Pacific Telecommunity. His research interests include leadership, radiocommunication systems, frequency spectrum management, ICT rules and regulations, spectrum monitoring, wireless networks and technologies. 